Marcelo Miqueletto

\title{
Desenvolvimento de procedimentos numéricos para análise de infiltração e estabilidade de taludes em bacias \\ de drenagem
}

Dissertação de Mestrado

Dissertação apresentada como requisito parcial para obtenção do título de Mestre pelo Programa de PósGraduação em Engenharia Civil da PUC-Rio.

Orientador: Eurípedes do Amaral Vargas Jr. 
Marcelo Miqueletto

\title{
Desenvolvimento de procedimentos numéricos para análise de infiltração e estabilidade de taludes em bacias \\ de drenagem
}

Dissertação apresentada como requisito parcial para obtenção do título de Mestre pelo Programa de PósGraduação em Engenharia Civil da PUC-Rio. Aprovada pela Comissão Examinadora abaixo assinada.

\author{
Eurípedes do Amaral Vargas Jr. \\ Presidente \\ PUC-Rio \\ Tácio Mauro Pereira de Campos \\ PUC-Rio
}

Nelson Ferreira Fernandes
UFRJ

Prof. José Eugênio Leal

Coordenador Setorial do Centro Técnico Científico - PUC-Rio 
Todos os direitos reservados. É proibida a reprodução total ou parcial do trabalho sem autorização da universidade, do autor e do orientador.

\section{Marcelo Miqueletto}

Graduou-se em Engenharia Civil pela Universidade Federal do Paraná. Durante a gradução desenvolveu trabalhos de iniciação científica e estagiou na área de goetecnia. Atuamente trabalha como engenheiro geotécnico em projetos de aproveitamentos hidrelétricos.

Miqueletto, Marcelo

Desenvolvimento de procedimentos numéricos para análise de infiltração e estabilidade de taludes em bacias de drenagem / Marcelo Miqueletto ; orientador: Eurípedes do Amaral Vargas Jr. - 2007.

152 f. : il. ; $30 \mathrm{~cm}$

Dissertação (Mestrado em Engenharia Civil)Pontifícia Universidade Católica do Rio de Janeiro, Rio de Janeiro, 2007.

Inclui bibliografia

1. Engenharia civil - Teses. 2. Fluxo saturado-não saturado. 3. Estabilidade de encostas. 4. Elementos finitos. I. Vargas Jr., Eurípedes do Amaral. II. Pontifícia Universidade Católica do Rio de Janeiro. Departamento de Engenharia civil. III. Título. 


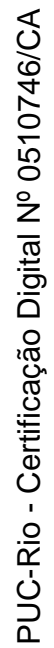

A meu pai. 


\section{Agradecimentos}

A meu pai, Primo, pelo amor e pelo exemplo de perseverança.

A minha madrinha e amiga, Malu, pelo amor e apoio.

Ao Professor Vargas, pela orientação, amizade e conhecimentos transmitidos durante a elaboração deste trabalho.

Ao André Muller que deu início a este trabalho e que muito ajudou no decorrer de sua elaboração.

Aos colegas e amigos da sala 317, em especial ao Julio, pelas inúmeras discussões e sugestões técnicas e filosóficas.

Aos habitantes permanentes e transientes da Frederico Eyer 121-C, em especial aos amigos João, Pedro e Thaís.

Aos amigos da PUC-Rio, pela amizade e convivência.

Aos amigos de Curitiba, sempre presentes e me apoiando em pensamento.

A todos os professores do Departamento de Engenharia Civil da PUC-Rio.

A todos os funcionários do Departamento de Engenharia Civil da PUC-Rio.

À PUC-Rio, à Capes e à FAPERJ pelo suporte a esta pesquisa, muito obrigado. 


\section{Resumo}

Marcelo Miqueletto. Desenvolvimento de procedimentos numéricos para análise de infiltração e estabilidade taludes em bacias de drenagem. Rio de Janeiro, 2007. 152p. Dissertação de Mestrado - Departamento de Engenharia Civil, Pontifícia Universidade Católica do Rio de Janeiro.

Este trabalho tem por objetivo o desenvolvimento de uma ferramenta numérica para avaliação do fluxo saturado-não saturado em encostas de grandes dimensões, com aplicação na análise de estabilidade dessas áreas. Emprega-se o método dos elementos finitos na solução da equação de Richards, considerando a carga de pressão como variável primária e utilizando formulação adequada para minimização dos problemas de conservação de massa, freqüentemente, associados a esse fato. $\mathrm{O}$ modelo constitutivo utilizado para a curva característica e função de condutividade hidráulica é o proposto por Van Genuchten (1980). Para solução da não-linearidade, emprega-se um método quasi-Newton (BFGS). Com o objetivo de minimizar os requisitos de memória computacional, utiliza-se esquema de armazenamento de matriz esparsa, associado ao método de gradiente biconjugado, na solução do sistema de equações. Paralelamente, é apresentado algoritmo de geração de malha tridimensional de elementos finitos, a partir de uma malha superficial de triângulos, representativa da topografia. Análises numéricas são executadas com a finalidade de validação do código gerado, comparando-se os resultados obtidos com aqueles gerados por outros programas já consagrados na

literatura técnica. É proposta metodologia para geração de mapas de susceptibilidade a escorregamentos translacionais rasos, empregando-se o método do talude infinito, associado à estrutura da malha de elementos finitos e aos resultados do problema de fluxo, incorporando-se, assim, o efeito do estado de não saturação na resistência do material.

\section{Palavras-chave}

Fluxo saturado-não saturado, Estabilidade de encostas, Elementos Finitos 


\section{Abstract}

Marcelo Miqueletto. Developement of numerical procedures for the analysis of infiltration and slope stability in catchment basins. Rio de Janeiro, 2007. 152p. MSc Dissertation - Departamento de Engenharia Civil, Pontifícia Universidade Católica do Rio de Janeiro.

The aim of this work is to develop a numerical tool for the analysis of saturated-unsaturated flow in large scale natural slopes, applied to the study of the stability of these areas. The finite element method is applied to solve the Richard's equation, taking into account the pressure head as the primary variable and using an adequate formulation to minimize the mass conservation issues. The constitutive model used to the characteristic curve and hydraulic constitutive function is the one presented by van Genuchten (1980). A quasi-Newton method (BFGS) is applied for the solution of the non-linearity. A sparse matrix storage scheme, with the objective of reducing the computational memory requirements, is associated to the bi-conjugated gradient method for the solution of the system of equations. An algorithm of finite elements mesh is presented, which generates the 3D mesh from a triangle superficial mesh representing the relief. Numerical analyses are performed in order to validate the code, by comparing the results with those generated by others widely known codes presented in the technical literature. A methodology for the generation of susceptibility maps to shallow translational landslides is delineated, which employs the infinite slope method to the finite elements mesh structure and the flow problem results, considering the effect of the unsaturated state in the material strength.

\section{Keywords}

Saturated-unsaturated flow, Slope stability, Finite element 


\section{Sumário}

$\begin{array}{ll}1 \text { Introdução } & 20\end{array}$

2 Solos não saturados 23

2.1. Fluxo em solos não saturados 23

2.1.1. Potencial da água no solo 23

2.1.2. Curva Característica 25

2.1.3. Lei de Darcy-Buckingham 29

2.1.4. Condutividade Hidráulica 32

2.1.5. Equação Richards

2.2. Resistência de solos não saturados $\quad 40$

2.3. Influência do fluxo em meios não-saturados na estabilidade de taludes 45

3 Implementações numéricas $\quad 48$

3.1. Solução Numérica da Equação de Richards 48

3.1.1. Formulação de Elementos Finitos 49

3.1.2. Discretização no tempo 56

3.1.3. Diagonalização da matriz de massa

3.1.4. Tratamento da Capacidade de Retenção Específica 59

3.1.5. Solução da não-linearidade $\quad 61$

3.1.6. Estimativa inicial do vetor de cargas de pressões nodais 67

3.1.7. Passo de tempo dinâmico $\quad 68$

$\begin{array}{ll}\text { 3.1.8. Critérios de convergência } & 70\end{array}$

3.1.9. Balanço de massa 72

3.1.10. Solução do sistema de equações 73

3.1.11. Gerador de malha $\quad 75$

3.2. Análise de estabilidade $\quad 77$

3.3. Implementação computacional $\quad 82$

4 Exemplos de validação $\quad 85$

4.1. Exemplos unidimensionais $\quad 85$

4.1.1. Fluxo unidimensional - Condição de contorno de Dirichlet. 85 
4.1.2. Fluxo unidimensional - Condição de contorno de Neumann e $\begin{array}{lr}\text { heterogeneidade do material. } & 92\end{array}$

4.2. Exemplos bidimensionais 98

4.2.1. Fluxo bidimensional - condição de contorno de Dirichlet. 98

4.2.2. Fluxo bidimensional - condição de contorno de Dirichlet - bulbo de $\begin{array}{lr}\text { infiltração. } & 102\end{array}$

4.2.3. Fluxo bidimensional - condição atmosférica (C.C. de Neumann e Dirichlet $\begin{array}{lr}\text { variáveis) - Talude. } & 106\end{array}$

$\begin{array}{ll}\text { 4.3. Exemplos tridimensionais } & 115\end{array}$

4.3.1. Fluxo tridimensional - condição de contorno de Dirichlet. 115

5 . Exemplo de aplicação 123

$\begin{array}{ll}\text { 5.1. Área de estudo } & 124\end{array}$

5.2. Propriedades dos materiais e parâmetros de análise 126

5.3. Resultados 131

6 Conclusões 142

$\begin{array}{ll}7 \text { Referências bibliográficas } & 145\end{array}$

8 Apêndices $\quad 151$

8.1. Modo de armazenamento de matriz esparsa indexado por linha $\quad 151$ 


\section{Lista de figuras}

Figura 1 - Curva característica 26

Figura 2 - Menisco de água no solo (Adaptado de Lu e Likos, 2004). 27

Figura 3 - Histerese 28

Figura 4 - Área útil de fluxo em meios porosos não saturados (Adaptado de Reichardt e Timm, 2004). 33

Figura 5 - Função de condutividade hidráulica. $\quad 34$

Figura 6 - Volume elementar de solo. 36

Figura 7 - Envoltória tridimensional de resistência para solos não saturados (adaptado de Lu e Likos, 2004). 43

Figura 8 - Não linearidade de $\phi^{b}$ (Adaptado de Lu e Likos, 2004). 44

Figura 9 - Elementos Finitos utilizados. (a) Elemento trilinear de 8 nós. (b)

Elemento trilinear de 6 nós.

Figura 10 - Malha tridimensional de elementos finitos paralepipédicos de 8 nós.

$\begin{array}{ll}\text { Figura } 11 \text { - Construção das linhas de nós, abaixo do nó de superfície. } & 76\end{array}$

Figura 12 - Diagrama de forças para o elemento 3D. 78

Figura 13 - Coluna de elementos 3D criada pelo gerador de malha. 80

$\begin{array}{ll}\text { Figura } 14 \text { - Seqüência de análise de estabilidade. } & 81\end{array}$

Figura 15 - Mapa de fator de segurança. $\quad 82$

Figura 16 - Fluxograma. $\quad 84$

$\begin{array}{ll}\text { Figura } 17 \text { - Curva característica. } & 87\end{array}$

Figura 18 - Curva de condutividade hidráulica. 88

Figura 19 - Carga de pressão em ponto situado a $15 \mathrm{~cm}$ de profundidade. $\quad 89$

Figura 20 - Volume acumulado. $\quad 90$

Figura 21- Perfis de infiltração.

Figura 22 - Carga de pressão em ponto situado a $15 \mathrm{~cm}$ de profundidade - opção

$1 . \quad 93$

Figura 23 - Carga de pressão em ponto situado a $45 \mathrm{~cm}$ de profundidade - opção

1. 93

Figura 24 - Perfis de infiltração - opção 1. 
Figura 25 - Carga de pressão em ponto situado a $15 \mathrm{~cm}$ de profundidade - opção 2.

Figura 26 - Carga de pressão em ponto situado a $45 \mathrm{~cm}$ de profundidade - opção 2.

Figura 27 - Perfis de infiltração - opção $2 . \quad 96$

Figura 28 - Curva característica. $\quad 98$

Figura 29 - Função de condutividade hidráulica. 99

Figura 30 - Malha de elementos finitos. 100

Figura 31 - Evolução da carga de pressão no tempo para o nó 33.

Figura 32 - Evolução da carga de pressão no tempo para o nó 51.

Figura 33 - Malha de elementos finitos. 103

Figura 34 - Evolução da carga de pressão no tempo para o nó 61. 104

Figura 35 - Evolução da carga de pressão no tempo para o nó 70. 104

Figura 36 - Bulbo de infiltração. 105

Figura 37 - Geometria simplificada para talude e malha de elementos finitos $\mathrm{RA}=10 . \quad 106$

Figura 38 - Malha de elementos finitos - RA=50. 108

Figura 39 - Evolução da carga de pressão para o nó A. 110

Figura 40 - Evolução da carga de pressão para o nó B. 110

Figura 41 - Evolução da carga de pressão para o nó C. 111

Figura 42 - Evolução da carga de pressão para o nó D. 111

Figura 43 - Evolução da carga de pressão para o nó E. 112

Figura 44 - Evolução da carga de pressão para o nó F. 112

Figura 45 - Evolução da carga de pressão para o nó G. 113

Figura 46 - Evolução da carga de pressão para o nó H. 113

Figura 47 - Malha de elementos finitos para as geometrias de taludes côncava (a e

b) e convexa (c e d). 116

Figura 48 - Evolução da carga de pressão para o nó 872, na geometria côncava.

Figura 49 - Evolução da carga de pressão para o nó 3781, na geometria côncava.

Figura 50 - Evolução da carga de pressão para o nó 2332, na geometria côncava. 
Figura 51 - Evolução da carga de pressão para o nó 1365, na geometria côncava.

Figura 52 - Evolução da carga de pressão para o nó 872, na geometria convexa.

Figura 53 - Evolução da carga de pressão para o nó 3781, na geometria convexa.

Figura 54 - Evolução da carga de pressão para o nó 2332, na geometria convexa.

Figura 55 - Evolução da carga de pressão para o nó 1365, na geometria convexa.

Figura 56 - Área de estudo. (Fonte: Fernades et al., 2001)

Figura 57 - Mapa de cicatrizes dos escorregamentos. (Fonte: Gomes, 2006) 126

Figura 58 - Curva característica adotada.

Figura 59 - Curva de condutividade hidráulica adotada.

Figura 60 - Precipitação diária para os 22 dias de simulação.

Figura 61 - Malha utilizada na análise.

Figura 62 - Mapa de distribuição de fator de segurança para o instante inicial. 133

Figura 63 - Mapa de cargas de pressão para o instante inicial.

Figura 64 - Mapa de distribuição de fator de segurança para T=180h (7,5 dias).

Figura 65 - Mapa de cargas de pressão para T=180h (7,5 dias).

Figura 66 - Mapa de distribuição de fator de segurança para T=288h (12dias). 135

Figura 67 - Mapa de cargas de pressão para T=288h (12dias).

Figura 68 - Mapa de distribuição de fator de segurança para T=480h (20dias). 136

Figura 69 - Mapa de cargas de pressão para T=480h (20dias).

Figura 70 - Mapa de distribuição de fator de segurança para T=480h (20dias) planta.

Figura 71 - Variação do FS, da carga de pressão e profundidade crítica, ao longo do tempo, para o nó de superfície 16160. Carga inicial de -0,5m.

138

Figura 72 - Variação do FS, da carga de pressão e profundidade crítica, ao longo do tempo, para o nó de superfície 10277. Carga inicial de -0,5m.

138

Figura 73 - Variação do FS, da carga de pressão e profundidade crítica, ao longo do tempo, para o nó de superfície 13257. Carga inicial de -0,5m. 
Figura 74 - Variação do FS, da carga de pressão e profundidade crítica, ao longo do tempo, para o nó de superfície 16160. Carga inicial de -5m.

Figura 75 - Variação do FS, da carga de pressão e profundidade crítica, ao longo do tempo, para o nó de superfície 10277. Carga inicial de -5m.

Figura 76 - Variação do FS, da carga de pressão e profundidade crítica, ao longo do tempo, para o nó de superfície 13257. Carga inicial de -5m. 


\section{Lista de tabelas}

Tabela 1 - Pontos de Gauss para elemento trilinear de 8 nós................................55

Tabela 2 - Pontos de Gauss para elemento trilinear de 6 nós................................. 55

Tabela 3 - Valores de infiltração imposta............................................................ 108

Tabela 4 - Tempos de análise........................................................................ 117

Tabela 5 - Precipitação diária para os 22 dias de simulação.............................. 128 


\section{Lista de símbolos}

\begin{tabular}{|c|c|}
\hline A & Área $\left[\mathrm{L}^{2}\right]$ \\
\hline$C(h)$ & Capacidade de retenção específica $\left[\mathrm{L}^{-1}\right]$ \\
\hline$C_{s}$ & Compressibilidade do esqueleto sólido $\left[\mathrm{M}^{-1} \mathrm{~T}^{2} \mathrm{~L}\right]$ \\
\hline$C_{w}$ & Compressibilidade da água $\left[\mathrm{M}^{-1} \mathrm{~T}^{2} \mathrm{~L}\right]$ \\
\hline$c^{\prime}$ & Coesão efetiva $\left[\mathrm{MT}^{-2} \mathrm{~L}^{-1}\right]$ \\
\hline$c^{*}$ & Coesão aparente $\left[\mathrm{MT}^{-2} \mathrm{~L}^{-1}\right]$ \\
\hline$c^{\prime \prime}$ & Coesão associada ao efeito da sucção $\left[\mathrm{MT}^{-2} \mathrm{~L}^{-1}\right]$ \\
\hline$\{f(h)\}$ & Função resíduo de iterações sucessivas $\left[\mathrm{L}^{3} \mathrm{~T}^{-1}\right]$ \\
\hline$\{f\}$ & Matriz Jacobiana ou matriz de iteração $\left[\mathrm{L}^{2} \mathrm{~T}^{-1}\right]$ \\
\hline $\mathrm{F}_{\mathrm{R}}$ & Força resistente $\left[\mathrm{MLT}^{-2}\right]$ \\
\hline $\mathrm{F}_{\mathrm{S}}$ & Força solicitante $\left[\mathrm{MLT}^{-2}\right]$ \\
\hline$F S$ & Fator de segurança $[-]$ \\
\hline$g$ & Aceleração da gravidade $\left[\mathrm{LT}^{-2}\right]$ \\
\hline$G$ & Densidade das partículas sólidas [-] \\
\hline$\{G\}$ & $\begin{array}{l}\text { Vetor associado aos gradientes de carga de elevação } \\
\text { nodais }\left[\mathrm{L}^{3} \mathrm{~T}^{-1}\right]\end{array}$ \\
\hline $\mathrm{h}$ & Carga de pressão [L] \\
\hline$\hat{h}(x, y, z, t)$ & Carga de pressão no interior do elemento finito [L] \\
\hline$h^{0}\left(x_{i}\right)$ & Valores de carga de pressão iniciais [L] \\
\hline $\bar{h}$ & Valores de carga de pressão imposta [L] \\
\hline $\mathrm{H}$ & Carga hidráulica total [L] \\
\hline$[H]$ & Matriz de condutividade $\left[\mathrm{L}^{2} \mathrm{~T}^{-1}\right]$ \\
\hline$i$ & Gradiente hidráulico [-] \\
\hline$[I]$ & Matriz identidade [-] \\
\hline$I_{\text {acum }}$ & $\begin{array}{l}\text { Volume acumulado de fluido que entrou ou saiu do } \\
\text { sistema }\left[\mathrm{L}^{3}\right]\end{array}$ \\
\hline$[J]$ & Matriz Jacobiana dos elementos [L] \\
\hline
\end{tabular}




\begin{tabular}{|c|c|}
\hline$|J|$ & Jacobiano $\left[\mathrm{L}^{3}\right]$ \\
\hline $\mathrm{k}$ & Coeficiente de permeabilidade intrínseca $\left[\mathrm{L}^{2}\right]$ \\
\hline $\mathrm{K}, \mathrm{K}(\theta), \mathrm{K}(\mathrm{h})$ & Condutividade hidráulica $\left[\mathrm{LT}^{-1}\right]$ \\
\hline$[\mathrm{K}],[\mathrm{K}(\theta)],[\mathrm{K}(\mathrm{h})], \mathrm{K}_{\mathrm{ij}}$ & Tensor de condutividade hidráulica $\left[\mathrm{LT}^{-1}\right]$ \\
\hline $\mathrm{K}_{\mathrm{s}}$ & Condutividade hidráulica saturada $\left[\mathrm{LT}^{-1}\right]$ \\
\hline$\left[\mathrm{K}_{\mathrm{s}}\right]$ & Tensor de condutividade hidráulica saturada $\left[\mathrm{LT}^{-1}\right]$ \\
\hline$l$ & Parâmetro de conectividade de poros de Mualen (1976) \\
\hline & {$[-]$} \\
\hline$m$ & Parâmetro do modelo de van Genuchten (1980) [-] \\
\hline$M B$ & Erro do balanço de massa [-] \\
\hline $\mathrm{n}$ & Parâmetro do modelo de van Genuchten (1980) [-] \\
\hline$N_{i}$ & Funções de interpolação [-] \\
\hline$q$ & Vazão específica $\left[\mathrm{L}^{2} \mathrm{~T}^{-1}\right]$ \\
\hline$\{q\}$ & Vetor de vazões específicas $\left[\mathrm{L}^{2} \mathrm{~T}^{-1}\right]$ \\
\hline$P$ & Peso total do bloco na análise de estabilidade $\left[\mathrm{MLT}^{-2}\right]$ \\
\hline Q & Vazão $\left[\mathrm{L}^{3} \mathrm{~T}^{-1}\right]$ \\
\hline$\{Q\}$ & Vetor de vazões nodais $\left[\mathrm{L}^{3} \mathrm{~T}^{-1}\right]$ \\
\hline $\mathrm{r}, \mathrm{s}, \mathrm{t}$ & Coordenadas locais dos elementos $[-]$ \\
\hline RA & Razão de aspecto [-] \\
\hline$R e$ & Número de Reynolds [-] \\
\hline$R$ & Resíduo do método de Galerkin $\left[\mathrm{L}^{3} \mathrm{~T}^{-1}\right]$ \\
\hline$s$ & Tensão tangencial solicitante $\left[\mathrm{MT}^{-2} \mathrm{~L}^{-1}\right]$ \\
\hline$S$ & Grau de saturação [-] \\
\hline$S_{s}$ & Armazenamento específico $\left[\mathrm{L}^{-1}\right]$ \\
\hline$[S]$ & Matriz de massa $\left[\mathrm{L}^{2}\right]$ \\
\hline $\mathrm{t}$ & Tempo $[\mathrm{T}]$ \\
\hline$u_{a}$ & Pressão do ar $\left[\mathrm{MT}^{-2} \mathrm{~L}^{-1}\right]$ \\
\hline$u_{w}$ & Pressão da água $\left[\mathrm{MT}^{-2} \mathrm{~L}^{-1}\right]$ \\
\hline$\{v\}$ & Vetor do método BFGS [-] \\
\hline$V$ & Volume total $\left[\mathrm{L}^{3}\right]$ \\
\hline$V_{w}$ & Volume de água $\left[\mathrm{L}^{3}\right]$ \\
\hline
\end{tabular}




\begin{tabular}{|c|c|}
\hline$V_{v}$ & Volume de vazios $\left[\mathrm{L}^{3}\right]$ \\
\hline$V_{s}$ & Volume de sólidos $\left[\mathrm{L}^{3}\right]$ \\
\hline$V^{e}$ & Volume do elemento $\left[\mathrm{L}^{3}\right]$ \\
\hline$\{w\}$ & Vetor do método BFGS [-] \\
\hline$W_{i}$ & Pesos de ponderação na integração de Gauss [-] \\
\hline$x_{i}$ & Coordenadas globais [L] \\
\hline $\mathrm{z}$ & Carga de elevação [L] \\
\hline$\alpha$ & Parâmetro do modelo de van Genuchten (1980) $\left[\mathrm{L}^{-1}\right]$ \\
\hline$\beta$ & Escalar multiplicador da busca linear no método BFGS \\
\hline & {$[-]$} \\
\hline$\chi$ & Parâmetro de tensão efetiva de Bishop [-] \\
\hline$\delta_{i j}$ & Delta de Kronecker \\
\hline$\{\delta\}$ & $\begin{array}{l}\text { Vetor de incremento de carga de pressão nodal entre } \\
\text { iterações no método BFGS [L] }\end{array}$ \\
\hline$\Delta t$ & Tamanho do passo de tempo $[\mathrm{T}]$ \\
\hline$\varepsilon^{2}$ & Erro de truncamento da série de Taylor \\
\hline$\phi^{\prime}$ & Ângulo de atrito efetivo [-] \\
\hline$\phi^{b}$ & $\begin{array}{l}\text { Parâmetro de resistência não saturada (Fredlung et al., } \\
\text { 1978) [-] }\end{array}$ \\
\hline$\gamma_{s}$ & Peso específico das partículas sólidas $\left[\mathrm{ML}^{-1} \mathrm{~T}^{-2}\right]$ \\
\hline$\gamma_{w}$ & Peso específico da água $\left[\mathrm{ML}^{-1} \mathrm{~T}^{-2}\right]$ \\
\hline$\{\gamma\}$ & $\begin{array}{l}\text { Vetor de incremento de vazão desiquilibrada nodal } \\
\text { entre iterações no método BFGS }\left[\mathrm{L}^{3} \mathrm{~T}^{-1}\right]\end{array}$ \\
\hline$\Gamma$ & Contorno do modelo \\
\hline$\Gamma_{D}$ & Contorno com condição de Dirichlet \\
\hline$\Gamma_{N}$ & Contorno com condição de Neumann \\
\hline$\varphi$ & Ângulo de máxima declividade do elemento no cálculo \\
\hline & de estabilidade [-] \\
\hline$\mu$ & Viscosidade dinâmica da água $\left[\mathrm{ML}^{-1} \mathrm{~T}^{-1}\right]$ \\
\hline$v$ & Viscosidade cinemática da água $\left[\mathrm{L}^{2} \mathrm{~T}^{-1}\right]$ \\
\hline$\theta$ & Unidade volumétrica $\left[\mathrm{L}^{3} \mathrm{~L}^{-3}\right]$ \\
\hline
\end{tabular}




\begin{tabular}{|c|c|}
\hline$\theta_{r}$ & Unidade volumétrica residual $\left[\mathrm{L}^{3} \mathrm{~L}^{-3}\right]$ \\
\hline$\theta_{s}$ & Unidade volumétrica de saturação $\left[\mathrm{L}^{3} \mathrm{~L}^{-3}\right]$ \\
\hline$\Theta$ & Umidade volumétrica relativa $\left[\mathrm{L}^{3} \mathrm{~L}^{-3}\right]$ \\
\hline$\rho$ & Massa específica $\left[\mathrm{ML}^{-3}\right]$ \\
\hline$\rho_{w}$ & Massa específica da água $\left[\mathrm{ML}^{-3}\right]$ \\
\hline$\sigma$ & Tensão total $\left[\mathrm{MT}^{-2} \mathrm{~L}^{-1}\right]$ \\
\hline$\sigma^{\prime}$ & Tensão efetiva de Terzaghi $\left[\mathrm{MT}^{-2} \mathrm{~L}^{-1}\right]$ \\
\hline$\tau$ & Resistência ao cisalhamento $\left[\mathrm{MT}^{-2} \mathrm{~L}^{-1}\right]$ \\
\hline$\xi$ & Porosidade [-] \\
\hline$\psi$ & Potencial total da água $\left[\mathrm{ML}^{2} \mathrm{~T}^{-2}\right]$ \\
\hline$\psi_{g}$ & Potencial gravitacional da água $\left[\mathrm{ML}^{2} \mathrm{~T}^{-2}\right]$ \\
\hline$\psi_{m}$ & Potencial matricial da água $\left[\mathrm{ML}^{2} \mathrm{~T}^{-2}\right]$ \\
\hline$\psi_{o}$ & Potencial osmótico da água $\left[\mathrm{ML}^{2} \mathrm{~T}^{-2}\right]$ \\
\hline$\psi_{p}$ & Potencial de pressão da água $\left[\mathrm{ML}^{2} \mathrm{~T}^{-2}\right]$ \\
\hline$\psi_{t}$ & Potencial térmico da água $\left[\mathrm{ML}^{2} \mathrm{~T}^{-2}\right]$ \\
\hline$\Omega$ & Domínio do modelo \\
\hline$\{\nabla H\}$ & Vetor gradiente de carga total $[\mathrm{L}]$ \\
\hline$\{\nabla h\}$ & Vetor gradiente de carga de pressão [L] \\
\hline
\end{tabular}




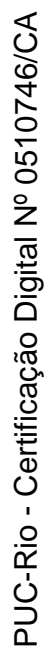

A rapadura é doce, mas não é mole não.

Sabedoria popular 